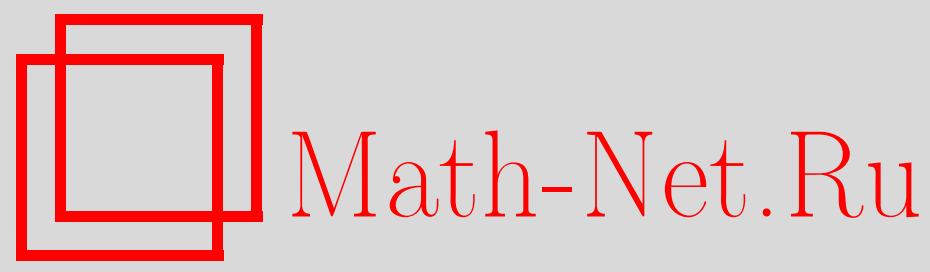

Г. И. Ивченко, С. А. Хонов, Е. А. Иванов, О точной оценке максимального правдоподобия состава расслоенной совокупности, Матем. заметки, 1997, том 62, выпуск 2, 216222

DOI: https://doi.org/10.4213/mzm1606

Использование Общероссийского математического портала Math-Net.Ru подразумевает, что вы прочитали и согласны с пользовательским соглашением http://www.mathnet.ru/rus/agreement

Параметры загрузки:

IP : 3.82 .47 .9

26 апреля 2023 г., 13:29:59 


\title{
О ТОЧНОЙ ОЦЕНКЕ МАКСИМАЛЬНОГО ПРАВДОПОДОБИЯ СОСТАВА РАССЛОЕННОЙ СОВОКУПНОСТИ
}

\author{
Г.И. Ивченко, С. А. Хонов, Е.А. Иванов
}

В заметке находится точный вид экстремальных значений целочисленного вектора $\bar{N}=\left(N_{1}, \ldots, N_{k}\right)$, максимизирующих функцию

$$
\prod_{j=1}^{k}\left(\begin{array}{c}
N_{j} \\
l_{j}
\end{array}\right)
$$

при произвольных целых $l_{1}>0, \ldots, l_{k}>0, k \geqslant 2$, и заданном $N^{0}=N_{1}+\cdots+N_{k}$, а также обсуждаются статистические применения этого резултата.

Библиограффия: 2 названия.

1. В приложениях математической статистики в ряде случаев приходится иметь дело с моделями, неизвестный параметр которых представляет собой целочисленный век$\operatorname{top} \bar{N}=\left(N_{1}, \ldots, N_{k}\right)$ с известным значением $N^{0}=N_{1}+\cdots+N_{k}$, входящий в выражение функции правдоподобия в виде сомножителя

$$
L(\bar{N} ; \bar{l})=\prod_{j=1}^{k}\left(\begin{array}{c}
N_{j} \\
l_{j}
\end{array}\right),
$$

называемого иногда ядром правдоподобия (здесь $\bar{l}=\left(l_{1}, \ldots, l_{k}\right)$ - наблюденное значение соответствующей достаточной статистики, и везде предполагается, что все $l_{j}>0$ и $\left.N^{0} \geqslant l=l_{1}+\cdots+l_{k}\right)$. В качестве примера можно привести задачу выборочного контроля, когда изделия классифицируются по $k \geqslant 2$ категориям качества. Для контроля партии из $N^{0}$ изделий, содержащей неизвестное число $N_{j}$ изделий $j$-го сорта, $j=1, \ldots, k$, из нее отбирается по схеме простой бесповторной выборки $n<N^{0}$ изделий. В этом случае достаточная статистика $\bar{\eta}=\left(\eta_{1}, \ldots, \eta_{k}\right)$, где $\eta_{j}$ - число изделий $j$-го сорта в выборке, имеет многомерное гипергеометрическое распределение

$$
P_{\bar{N}}(\bar{\eta}=\bar{l})=L(\bar{N} ; \bar{l})\left(\begin{array}{c}
N^{0} \\
n
\end{array}\right)^{-1}, \quad l_{1}+\cdots+l_{k}=n .
$$

Работапервого автора выполнена при финансовой поддержке Российского фонда фундаментальных исследований, грант № 97-01-00387. 
Задача состоит в том, чтобы по наблюдению над $\bar{\eta}$ оценить состав $\bar{N}=\left(N_{1}, \ldots, N_{k}\right)$ партии.

Вообще, с подобной ситуацией часто сталкиваются при выборочном обследовании расслоенных конечных совокупностей вида $U=U_{1} \cup \cdots \cup U_{k}, U_{i} \cap U_{j}=\varnothing, i \neq j$, когда размеры $N_{j}=\left|U_{j}\right|, j=1, \ldots, k$, составляющих ее слоев неизвестны и должны быть оценены по результатам соответствуюшего статистического эксперимента. Подобные задачи активно обсуждались в литературе: накоплено большое число результатов для различных схем получения данных (организации выборки) и различных методов оценивания неизвестного состава $\bar{N}=\left(N_{1}, \ldots, N_{k}\right)$ совокупности.

Обзор этих результатов и соответствующую библиографию можно найти в последних работах на эту тему [1], [2].

Настоящая заметка посвящена решению одной из открытых задач в этой области. Мы рассматриваем оценивание параметра $\bar{N}$ по принщипу правдоподобия, которьй, как отмечается в [1], с различных позиций является в данной проблематике более предпочтительным и согласно которому в качестве оценки параметра $\bar{N}$ выбирается точка максимума ядра правдоподобия $L(\bar{N} ; \bar{l})$. Таким образом, по определению оценка максимального правдоподобия (ОМП) $\widehat{\bar{N}}=\left(\widehat{N}_{1}, \ldots, \widehat{N}_{k}\right)$ параметра $\bar{N}$ определяется в данном случае как решение следуюшей дискретной задачи на условньй экстремум:

$$
\widehat{\bar{N}}=\underset{\bar{N} \in \mathcal{N}}{\arg \max } L(\bar{N} ; \bar{l}), \quad \mathscr{N}=\left\{\bar{N}: N_{1}+\cdots+N_{k}=N^{0}\right\} .
$$

Специфика задачи (1) заключается в том, что здесь мы имеем дело с функциями от многомерного целочисленного аргумента, для которых отсутствуют обшие методы решения подобных экстремальньх задач. Лишш для простейшего случая $k=2$ (ядро $L$ является функцией фактически одного переменного $N_{1}$ ) решение задачи (1) достаточно просто и известно из литературы: $\widehat{N}_{1}=\left[\left(N^{0}+1\right) l_{1} / l\right]$, где $[\cdot]$ есть целая часть числа; при этом, если число $\left(N^{0}+1\right) l_{1} / l$ целое, то $\widehat{N}_{1}-1$ также является ОМП параметра $N_{1}$. Для общего случая $k \geqslant 2$ явный вид решений задачи (1) дается нами впервые. Мы также выясняем степень неоднозначности ОМП $\widehat{\bar{N}}$ и приводим простой алгоритм для ее компютерных вычислений.

2. Ключевую роль в дальнейшем играет функция

$$
F(\lambda)=\sum_{j=1}^{k}\left[\lambda l_{j}\right], \quad \lambda \geqslant 0 .
$$

Свойства этой функции детально обсуждаются в п. 3, здесь мы отметим лишь некоторые из них, необходимые для формулировки и доказательства основного результата заметки: $F(\lambda)$ - неубывающая, непрерывная справа ступенчатая целочисленная функция, точки скачков которой кратны обратным значениям параметров $l_{j}$, для нее однозначно определена обратная функция

$$
F^{-1}(N)=\min \{\lambda: F(\lambda) \geqslant N\}, \quad N=1,2, \ldots,
$$

множество значений которой совпадает с множеством точек роста $F$. Введем также множество $J(\lambda)=\left\{j: \lambda l_{j}\right.$-целое $\}$, т.е. множество номеров тех слагаемых в $(2)$, которые 
в точке $\lambda$ претерпевают единичньй скачок. Тогда величина скачка функции $F$ в точке $\lambda$ есть

$$
\Delta F(\lambda)=F(\lambda)-F(\lambda-0)=|J(\lambda)| .
$$

Пусть теперь $\lambda^{0}=F^{-1}\left(N^{0}\right)$ и $J^{0}=J\left(\lambda^{0}\right)$, т.е. $\lambda^{0}$ - точка скачка $F$, определяемая из условия $F\left(\lambda^{0}-0\right)<N^{0} \leqslant F\left(\lambda^{0}\right)$, а $\left|J^{0}\right|$ - величина этого скачка. Отметим, что $\lambda^{0} \geqslant 1\left(\right.$ так как $\left.N^{0} \geqslant l\right)$, причем $\lambda^{0}=1$ тогда и только тогда, когда $N^{0}=l$, a $\left|J^{0}\right| \leqslant k$. Наконец, положим $\delta^{0}=F\left(\lambda^{0}\right)-N^{0}$, тогда $0 \leqslant \delta^{0}<\left|J^{0}\right|$.

Теорема. Решения задачи (1) имеют вид

$$
\widehat{\bar{N}}=\left(\left[\lambda^{0} l_{j}\right]-I_{D}(j), j=1, \ldots, k\right),
$$

где $D$ - произвольное подмнохсество размера $\delta^{0}$ множества $J^{0}$ и $I_{D}(j)-$ его индикатор.

Прежде чем доказьвать теорему, дадим некоторые пояснения. Множество $J^{0}$ по определению всегда непусто, а параметр $\delta^{0}$ может быть равен 0, и тогда $D=\varnothing$. Лиш в этом случае, т.е. при $F\left(\lambda^{0}\right)=N^{0}$, ОМП $\widehat{\bar{N}}$ определяется однозначно: $\widehat{\bar{N}}=\left(\left[\lambda^{0} l_{j}\right]\right.$, $j=1, \ldots, k)$. В противном случае, т.е. при $\delta^{0}>0$, существует

$$
\left(\begin{array}{c}
\left|J^{0}\right| \\
\delta^{0}
\end{array}\right)
$$

различных вариантов выбора подмножества $D \subset J^{0}$ и, следовательно, соответствующих версий ОМП. Таким образом, в рассматриваемой задаче ОМП определяется, вообще говоря, неоднозначно, что характерно для моделей с дискретным параметром. Однако, неоднозначность здесь имеет специфический характер: различные версии ОМП отличаются лиш изменением на 1 некоторых координат. Наконец, предположение, что все $l_{j}>0$, не является ограничением общности задачи, так как в противном случае (но при $l>0)$ лишь понижается размерность вектора $\bar{N}$, поскольку функция $L(\bar{N} ; \bar{l})$ фактически зависит от $N_{j}$ или $l_{j}>0$. В терминах же ОМП это означает, что при $l_{j}=0$ полагается $\widehat{N}_{j}=0$.

ДоКАЗАТЕЛЬСТво ТЕОРЕМЫ. По определению ОМП $\widehat{\bar{N}}$ есть такая точка гиперплоскости $\mathscr{N}($ см. (1)), в которой вьполняется неравенство $L(\bar{N}+\bar{\delta} ; \bar{l}) \leqslant L(\bar{N} ; \bar{l})$, где $\bar{\delta}=$ $\left(\delta_{1}, \ldots, \delta_{k}\right)$ - произвольный ненулевой целочисленный вектор, удовлетворяющий условиям $\delta_{1}+\cdots+\delta_{k}=0$ и $N_{j}+\delta_{j} \geqslant l_{j}$ для всех $j$. Обозначив $J_{-} \equiv\left\{j: \delta_{j}<0\right\}$, $J_{+}=\left\{j: \delta_{j}>0\right\}$, имеем

$$
\begin{aligned}
& \frac{L(\bar{N}+\bar{\delta} ; \bar{l})}{L(\bar{N} ; \bar{l})}=\prod_{j \in J_{-}} \frac{\left(N_{j}+\delta_{j}+1-l_{j}\right) \cdots\left(N_{j}-l_{j}\right)}{\left(N_{j}+\delta_{j}+1\right) \cdots N_{j}} \prod_{j \in J_{+}} \frac{\left(N_{j}+1\right) \cdots\left(N_{j}+\delta_{j}\right)}{\left(N_{j}+1-l_{j}\right) \cdots\left(N_{j}+\delta_{j}-l_{j}\right)} \\
& \quad=\prod_{j \in J_{-}}\left(1-\frac{l_{j}}{N_{j}+\delta_{j}+1}\right) \cdots\left(1-\frac{l_{j}}{N_{j}}\right) / \prod_{j \in J_{+}}\left(1-\frac{l_{j}}{N_{j}+1}\right) \cdots\left(1-\frac{l_{j}}{N_{j}+\delta_{j}}\right) \\
& \quad \leqslant \prod_{j \in J_{-}}\left(1-\frac{l_{j}}{N_{j}}\right)^{\left|\delta_{j}\right|} / \prod_{j \in J_{+}}\left(1-\frac{l_{j}}{N_{j}+1}\right)^{\delta_{j}} .
\end{aligned}
$$


$\mathrm{B}$ частности, если все ненулевые $\delta_{j}= \pm 1$, то в последнем неравенстве имеет место знак равенства, и рассматриваемое отношение будет не больше 1 тогда и только тогда, когда выполняется условие

$$
\frac{l_{j}}{N_{j}+1} \leqslant \frac{l_{i}}{N_{i}}, \quad i \neq j
$$

Так как

$$
\sum_{j \in J_{-}}\left|\delta_{j}\right|=\sum_{j \in J_{+}} \delta_{j}
$$

то точка $\bar{N}$, удовлетворяющая (4), будет точкой и глобального максимума функции $L(\bar{N} ; \bar{l})$. Тем самым, любое решение неравенств $(4)$, принадлежащее гиперплоскости $\mathcal{N}$, является ОМП $\widehat{\bar{N}}$.

Проверим теперь, что любая точка из указанных в (3), является ОМП, т.е. для нее выполняется (4) и она лежит на $\mathscr{N}$. Для индексов $j \in J^{0}$ величины $\lambda^{0} l_{j}$ являются целыми числами, поэтому в (3)

$$
\widehat{N}_{j}= \begin{cases}\lambda^{0} l_{j}-1 & \text { при } j \in D, \\ \lambda^{0} l_{j} & \text { при } j \in J^{0} \backslash D .\end{cases}
$$

Если $j \notin J^{0}$, то $\lambda^{0} l_{j}-1<\widehat{N}_{j}=\left[\lambda^{0} l_{j}\right]<\lambda^{0} l_{j}$. Таким образом, в любом случае (для любого $j$ ) $\lambda^{0} l_{j}-1 \leqslant \widehat{N}_{j} \leqslant \lambda^{0} l_{j}$ или $\widehat{N}_{j} \leqslant \lambda^{0} l_{j} \leqslant \widehat{N}_{j}+1$. Отсюда имеем $\widehat{N}_{j} / l_{j} \leqslant \lambda^{0} \leqslant\left(\widehat{N}_{j}+1\right) / l_{j}$ для любого $j$, и значит, для $\widehat{\widehat{N}}=\left(\widehat{N}_{1}, \ldots, \widehat{N}_{k}\right)$ также выполняются неравенства (4). Наконец, тот факт, что точка $\widehat{\bar{N}}$ лежит на гиперплоскости $\mathscr{N}$, следует из очевидных равенств

$$
\sum_{j=1}^{k} \widehat{N}_{j}=F\left(\lambda^{0}\right)-\sum_{j=1}^{k} I_{D}(j)=N^{0}+\delta^{0}-|D|=N^{0} .
$$

Обратно, покажем, что любое решение системы (4), принадлежащее $\mathcal{N}$, может быть представлено в виде (3). Пусть точка $\bar{N} \in \mathcal{N}$ удовлетворяет соотношениям (4). Обозначим

$$
\lambda^{\prime}=\max _{j} \frac{N_{j}}{l_{j}}, \quad E=\left\{j: \frac{N_{j}}{l_{j}}=\lambda^{\prime}\right\}
$$

и убедимся сначала, что $\lambda^{\prime}=\lambda^{0}$.

Во-первых, для любого $j$ имеем $N_{j} \leqslant \lambda^{\prime} l_{j}$, т.е. $N_{j} \leqslant\left[\lambda^{\prime} l_{j}\right]$, следовательно, $N^{0} \leqslant F\left(\lambda^{\prime}\right)$. Во-вторых, для $j \in E$ имеем $N_{j}=\lambda^{\prime} l_{j}$, а в силу (4) $\lambda^{\prime} l_{j} \leqslant N_{j}+1$. Отсюда следует, что для любого $\lambda<\lambda^{\prime}\left[\lambda l_{j}\right] \leqslant \lambda l_{j}<N_{j}$ при $j \in E$ и $\left[\lambda l_{j}\right] \leqslant N_{j}$ при всех $j$. Поскольку множество $E$ непусто, отсюда получаем соотношение $F(\lambda)<N^{0}$. Таким образом, $F(\lambda)<N^{0} \leqslant F\left(\lambda^{\prime}\right)$ для любого $\lambda<\lambda^{\prime}$, т.е. $\lambda^{\prime}=F^{-1}\left(N^{0}\right)=\lambda^{0}$.

Положим теперь $D=\left\{j: \lambda^{0} l_{j}=N_{j}+1\right\}$. Ясно, что $D \subset J^{0}$ ипри $j \notin D\left[\lambda^{0} l_{j}\right] \leqslant N_{j}$, что с учетом полученного вьше неравенства $N_{j} \leqslant\left[\lambda^{\prime} l_{j}\right]$ дает равенство $N_{j}=\left[\lambda^{0} l_{j}\right]$. Таким образом, имеем представление типа (3):

$$
N_{j}=\left\{\begin{array}{ll}
\lambda^{0} l_{j}-1 & \text { при } j \in D, \\
{\left[\lambda^{0} l_{j}\right]} & \text { при } j \notin D,
\end{array}=\left[\lambda^{0} l_{j}\right]-I_{D}(j), \quad j=1, \ldots, k,\right.
$$

из которого также следует, что $N^{0}=F\left(\lambda^{0}\right)-|D|$, т.е. $|D|=F\left(\lambda^{0}\right)-N^{0}=\delta^{0}$. Теорема доказана.

К вычислительным аспектам, связанньм с формулами (3), мы вернемся в п. 4. 
3. Для практического использования результата (3) нужно иметь удобный алгоритм вычисления параметра $\lambda^{0}=F^{-1}\left(N^{0}\right)$, т.е. алгоритм обращения функции $F$, определенной в (2). В данном пункте мы проводим более детальный анализ этой функции и получаем ее конструктивное описание, которое позволяет построить эффективный и удобный для компюютерной реализации алгоритм.

Введем две вспомогательные функции, первая из которых для любого $\lambda \geqslant 0$ вычисляет ту точку скачка, в которой функция $F$ впервые становится равной $F(\lambda)$ :

$$
p(\lambda)=\min \left\{\lambda^{\prime}: F\left(\lambda^{\prime}\right)=F(\lambda)\right\},
$$

а вторая вычисляет ближайшую после $\lambda$ точку скачка $F$ :

$$
n(\lambda)=\min \left\{\lambda^{\prime}: F\left(\lambda^{\prime}\right)>F(\lambda)\right\}
$$

Таким образом, вместе эти две функции определяют полуинтервал постоянства функции $F$, которому принадлежит точка $\lambda: F\left(\lambda^{\prime}\right)=F(\lambda)$ тогда и только тогда, когда $\lambda^{\prime} \in[p(\lambda), n(\lambda))$.

Для практического вычисления этих функций имеются удобные формулы:

$$
p(\lambda)=\max _{j} \frac{\left[\lambda l_{j}\right]}{l_{j}}, \quad n(\lambda)=\min _{j} \frac{\left[\lambda l_{j}\right]+1}{l_{j}} .
$$

Действительно, так как всегда $\left[\lambda l_{j}\right] \leqslant \lambda l_{j}<\left[\lambda l_{j}\right]+1$, имеют место неравенства

$$
\frac{\left[\lambda l_{j}\right]}{l_{j}} \leqslant p(\lambda) \leqslant \lambda<n(\lambda) \leqslant \frac{\left[\lambda l_{j}\right]+1}{l_{j}} .
$$

Отсюда, во-первых, имеем $\left[\lambda l_{j}\right] \leqslant p(\lambda) l_{j} \leqslant \lambda l_{j}$, т.е. $\left[p(\lambda) l_{j}\right]=\left[\lambda l_{j}\right]$ и, следовательно, $F(p(\lambda))=F(\lambda)$.

Во-вторьх, для любого $\lambda^{\prime} \in[\lambda, n(\lambda))$ вьполняются соотношения $\lambda l_{j} \leqslant \lambda^{\prime} l_{j}<\left[\lambda l_{j}\right]+1$, откуда также $\left[\lambda^{\prime} l_{j}\right]=\left[\lambda l_{j}\right]$ и, следовательно, $F\left(\lambda^{\prime}\right)=F(\lambda)$. Таким образом, на полуинтервале $[p(\lambda), n(\lambda))$ функция $F$ постоянна и равна $F(\lambda)$. В то же время, как $p(\lambda)$ (при $\lambda \geqslant\left(\max _{j} l_{j}\right)^{-1}$ ), так и $n(\lambda)$ (при любом $\lambda \geqslant 0$ ) являются точками скачков функции $F$, поскольку они кратны обратньм величинам некоторых $l_{j}$. При этом величина скачка $|J(n(\lambda))|$ в точке $n(\lambda)$ вычисляется по формуле

$$
|J(n(\lambda))|=\left|\left\{j: \frac{\left[\lambda l_{j}\right]+1}{l_{j}}=n(\lambda)\right\}\right|,
$$

и, аналогично, в точке $p(\lambda)$

$$
|J(p(\lambda))|=\left|\left\{j: \frac{\left[\lambda l_{j}\right]}{l_{j}}=p(\lambda)\right\}\right| .
$$

Действительно, например, $J(n(\lambda))$ - это по определению множество тех $j$, для которых $n(\lambda) l_{j}$ - целое, т.е. в него входят указанные в (6) значения $j$. Для остальных $j$ в силу (5) $\lambda l_{j}<n(\lambda) l_{j}<\left[\lambda l_{j}\right]+1$, т.е. $n(\lambda) l_{j}$ не может быть целым числом. Таким 
образом, $J(n(\lambda))$ включает лишь указанные в (6) значения $j$. Аналогично проверяется и (7).

Роль функции $n(\lambda)$ не исчерпьвается сказанным вьше. Эта функция обладает важным свойством "динамики” : поскольку $n(\lambda)$ всегда вычисляет непосредственно следующую за $\lambda$ точку скачка функции $F$, то с ее помощью можно последовательно перечислить все точки скачков на любом интервале $\lambda>\lambda_{0} \geqslant 0$. Именно, это будет последовательность $\left\{\lambda_{t}=n\left(\lambda_{t-1}\right)=n_{t}\left(\lambda_{0}\right), t=1,2, \ldots\right\}$; здесь $n_{t}$ есть $t$-я итерация функции $n$ : $n_{t}(\lambda)=n\left(n_{t-1}(\lambda)\right), n_{0}(\lambda)=\lambda$. Последовательность $\left\{\left|J\left(\lambda_{t}\right)\right|, t=1,2, \ldots\right\}$ (см. (6)) задает величины соответствующих скачков. Тем самым, функцию $F(\lambda)$ при $\lambda \geqslant \lambda_{0}$ можно конструктивно описать с помощью двойной последовательности $\left\{\left(\lambda_{t},\left|J\left(\lambda_{t}\right)\right|\right), t=\right.$ $1,2, \ldots\}$ :

$$
F(\lambda)=F\left(\lambda_{0}\right)+\sum_{i=1}^{t}\left|J\left(\lambda_{i}\right)\right|, \quad \lambda \in\left[\lambda_{t}, \lambda_{t+1}\right), \quad t=0,1,2, \ldots
$$

(пустая сумма при $t=0$ здесь заменяется на 0 ). В частности, при $\lambda_{0}=0$ формула (8) дает конструктивное представление $F$ на всей полупрямой $\lambda \geqslant 0$.

Наконец, представление (8) позволяет предложить следуюший простой алгоритм вычисления обратной функции $F^{-1}(N)$ для любого целого $N \geqslant F\left(\lambda_{0}\right)$ (а при $\lambda=0$ - для любого $N=1,2, \ldots)$ : при $N>F\left(\lambda_{0}\right)$

$$
F^{-1}(N)=\lambda_{\tau(N)}, \quad \text { где } \tau(N)=\min \left\{t: F\left(\lambda_{t}\right) \geqslant N\right\},
$$

если же $N=F\left(\lambda_{0}\right)$, то $F^{-1}(N)=p\left(\lambda_{0}\right)$.

ЗАмЕчАнИЕ. Если требуется вычислить значение обратной функции $F^{-1}$ лишш в отдельной конкретной точке $N^{0}$ (как, например, в формулах $(3)$ ), то общий алгоритм (9) можно несколько усовершенствовать, если учесть, что при $\lambda_{0}=\left(N^{0}+1\right) / l$ имеют место оценки

$$
N^{0}+1-k<F\left(\lambda_{0}\right)=\sum_{j=1}^{k}\left[\frac{N^{0}+1}{l} l_{j}\right] \leqslant N^{0}+1 .
$$

Здесь $F\left(\lambda_{0}\right)=N^{0}+1$ тогда и только тогда, когда $J\left(\lambda_{0}\right)=\{1,2, \ldots, k\}$, т.е. $\lambda_{0}-$ точка скачка и $\Delta F\left(\lambda_{0}\right)=\left|J\left(\lambda_{0}\right)\right|=k$, следовательно, в этом случае $F^{-1}\left(N^{0}\right)=\lambda_{0}$. Если же $F\left(\lambda_{0}\right) \leqslant N^{0}$, то "работает" общий алгоритм (9), при этом неравенство $F\left(\lambda_{0}\right)<N^{0}$ возможно лишь при $k>2$, и в этом случае число шагов алгоритма не превосходит $N^{0}-F\left(\lambda_{0}\right) \leqslant k-2\left(t\right.$-м шагом алгоритма является выгисление $\lambda_{t}=n\left(\lambda_{t-1}\right)$ и сравнение $F\left(\lambda_{t}\right)$ с $\left.N^{0}, t=1,2, \ldots\right)$.

4. Вернемся к нашей основной задаче и применим изложенную в п. 3 методику для вычисления ОМП (3). Как отмечено в п. 2, при $N^{0}=l$ параметр $\lambda^{0}=F^{-1}\left(N^{0}\right)=1$ и, следовательно, $\widehat{\bar{N}}=\bar{l}$ (что, конечно, очевидно). Поэтому далее будем считать, что $N^{0}>l$.

Применяя усовершенствованньй (в соответствии с замечанием) вариант алгоритма (9), получим, что при $F\left(\lambda_{0}\right)=N^{0}+1$ параметры $\lambda^{0}=\lambda_{0}=\left(N^{0}+1\right) / l, \delta^{0}=$ $F\left(\lambda_{0}\right)-N^{0}=1$, и поэтому имеется $k$ версий ОМП: $\overline{\bar{N}}_{i}=\lambda_{0} \bar{l}-\bar{e}_{i}, i=1, \ldots, k$, где $\bar{e}_{i}-$ $i$-й единичный вектор. 
В случае $F\left(\lambda_{0}\right)=N^{0}$ согласно (9) $\lambda^{0}=p\left(\lambda_{0}\right), \delta^{0}=0$, и по формуле (3) ОМП определяется однозначно: $\widehat{\bar{N}}=\left(\left[p\left(\lambda_{0}\right) l_{j}\right]=\left[\lambda_{0} l_{j}\right], j=1, \ldots, k\right)$. При $k=2$ этими двумя вариантами все исчерпьвается, и мы имеем (указанньй в п. 1) известньй результат.

Если же $k>2$ (что представляет для нас наибольший интерес), то возможен также случай $F\left(\lambda_{0}\right)<N^{0}$, и здесь в соответствии с (9) $\lambda^{0}=\lambda_{\tau^{0}}$, где $\tau^{0}=\min \left\{t: F\left(\lambda_{t}\right) \geqslant N^{0}\right\}$. Тем самым, вместе с (3) мы имеем эффективно реализуемый (за число шагов, не превосходящее $k-2$ ) конструктивный алгоритм вычисления всех возможных значений ОМП.

ПРИмЕР. Пусть $k=3, N^{0}=14$ и $\bar{l}=(3,1,4)$. Здесь $\lambda_{0}=15 / 8, F\left(\lambda_{0}\right)=13$, т.е. имеем случай $F\left(\lambda_{0}\right)<N^{0}$. Согласно алгоритму (9) находим $\lambda_{1}=n\left(\lambda_{0}\right)=2, F\left(\lambda_{1}\right)=16$, откуда $\lambda^{0}=F^{-1}\left(N^{0}\right)=\lambda_{1}$ и $\delta^{0}=2$. Далее, $J^{0}=\{1,2,3\}$ и подмножество $D$ в теореме может быть выбрано тремя способами: $\{1,2\},\{1,3\}$ и $\{2,3\}$. Поэтому имеем три версии ОМП: $(5,1,8),(5,2,7)$ и $(6,1,7)$.

\section{СПИСОК ЦИТИРОВАННОЙ ЛИТЕРАТУРЫ}

[1] Ivchenko G. I., Khonov S. A. Maximum likelihood estimation for a stratified finite population // Math. Methods Statist. 1994. V. 3. № 4. P. 346-361.

[2] Ивченко Г.И., Хонов С. А. Статистическое оценивание состава конечной совокупности // Дискретная матем. 1996. Т. 8. №1. С. 3-40.

Московский государственный институт электроники и математики 